60-61| 2018

La culture dans l'enseignement du français langue étrangère: conceptions théoriques, programmes et manuels auX $\mathrm{XIX}^{\mathrm{e}}$ et $\mathrm{XX}^{\mathrm{e}}$ siècles

\title{
La composante culturelle dans la construction de la discipline français langue étrangère (XVIII $)$
}

The cultural component in the construction of the discipline of French as a foreign language (18th century)

Javier Suso López

\section{OpenEdition}

\section{Édition électronique}

URL : https://journals.openedition.org/dhfles/4982

DOI : $10.4000 /$ dhfles.4982

ISSN : 2221-4038

Éditeur

Société Internationale pour l'Histoire du Français Langue Étrangère ou Seconde

Édition imprimée

Date de publication : 1 janvier 2018

Pagination : 63-84

ISSN : 0992-7654

Référence électronique

Javier Suso López, « La composante culturelle dans la construction de la discipline français langue étrangère (XVIIIe) », Documents pour l'histoire du français langue étrangère ou seconde [En ligne], 60-61 2018, mis en ligne le 01 juin 2019, consulté le 26 mars 2023. URL : http://journals.openedition.org/ dhfles/4982 ; DOI : https://doi.org/10.4000/dhfles.4982

Ce document a été généré automatiquement le 26 mars 2023.

Tous droits réservés 


\section{La composante culturelle dans la construction de la discipline français langue étrangère $\left(\mathrm{XVIII}^{\mathrm{e}}\right)^{1}$}

The cultural component in the construction of the discipline of French as a foreign language (18th century)

Javier Suso López

Marie-Christine Kok Escalle \& Madeleine Van Strien-Chardonneau concluent leur étude sur le métier de maître de langue en Hollande aux XVII ${ }^{\mathrm{e}}$-XIX ${ }^{\mathrm{e}}$ siècles en affirmant que « dans les manuels destinés à l'apprentissage de la langue française se trouvent [...] un savoir linguistique et culturel (histoire, géographie, coutumes) sur le monde de l'autre, dans lequel l'apprenant est inclus par contraste ou par association (les normes sociale et morale étant par exemple similaires) » et que le maitre de langues «enseigne une discipline, mais son action porte plus généralement sur l'éducation et l'instruction de ses élèves. Le maître de langues [est un] passeur entre les deux langues, entre les deux cultures $[. .] ».(2005: 33 / 34,143)$; il joue ainsi, outre son rôle d'enseignant de langue, un rôle de "médiateur social, éducatif et culturel » (ibid., 136). Nous désirons dans cette étude ${ }^{2}$ montrer que cette conclusion est généralisable à l'enseignement du français comme langue étrangère dans l'ensemble des pays européens, et montrer que cette conception de la matière constitue l'image de marque qui préside à sa disciplinarisation.

\section{Le français : langue utile, langue d'agrément, langue de l'éducation de tout lettré et de toute personne bien élevée (XVIe-XVIII ${ }^{e}$ siècles)}

Disons tout d'abord que la conception de la langue française, tout au long du XVI ${ }^{e}, \mathrm{XVII}^{\mathrm{e}}$ mais surtout au XVIII ${ }^{e}$ siècle, était très diverse en fonction des contextes où elle était enseignée ou des modalités ou elle était apprise, et donc des usages présumés ou réels 
qui s'offraient aux apprenants. Le français était, tout d'abord, une langue dotée d'une "utilité professionnelle pour le commerce ou la diplomatie, utilité pratique pour le voyageur... » (Argaud \& Kok Escalle 2012 : 111). Cette conception pratique des usages du français, prônée par la bourgeoisie marchande dès le XVI ${ }^{\mathrm{e}}$ siècle ( $c f$. par exemple les ouvrages de Gabriel Meurier, ou bien les écoles de français de Peeter Heyns aux PaysBas dans Swiggers 2009 : 102-103), constitue une manière d'envisager le français comme langue vivante éminemment utile.

3 Ce concept utilitaire du FLE (la maîtrise du code linguistique) se double déjà d'une approche qui ne concerne pas uniquement l'aspect linguistique; les dialogues, insérés dans les grammaires ou bien édités à part mais composés comme matériel d'enseignement complémentaire aux grammaires ${ }^{3}$, mettent en place de nombreuses observations sur les modes de vie et les mœurs des Français (mais aussi, des étrangers qui apprennent la langue française, par comparaison), mais aussi sur la réalité sociopolitique de l'époque. The true Advancement of the French tongue, de Claude Mauger (1653) introduit une description complète du château de Versailles, puis un voyage pour visiter les châteaux de Touraine ; rentrés à Paris, les élèves 'goûtent' des différents types de pains faits de Paris... Sa Grammaire $\left({ }^{11} 1684\right)^{4}$ comprend une liste de phrases familières (sous forme de dialogues entre une jeune demoiselle et sa gouvernante: 191-204), mais aussi onze dialogues (205-337) où les sujets d'entretien comprennent des questions d'actualité (l'état de la France sous Louis XIV, la guerre contre les Turcs), des questions culturelles (les monuments de Rome, la philosophie, la nature des plantes, le soleil), des formules utiles à la communication et des questions galantes (l'amour, 'lequel des deux sexes, de l'homme \& de la femme, est le plus noble')... Le culturel imprègne ainsi, les dialogues, les listes de vocabulaire (Argaud \& Kok Escalle rappellent le concept de 'lexiculture' mis en relief par Galisson), et les grammaires des XVI ${ }^{\mathrm{e}}$ et XVII ${ }^{\mathrm{e}}$ siècles :

les contenus culturels [...] sont inévitablement présents dans tous les supports pédagogiques et [...] le culturel imprègne le document le plus simple sous des formes multiples, que ce soit les textes écrits, religieux ou profanes, littéraires ou familiers, les fables, les contes, les histoires, les lettres, les entretiens, les anecdotes et les proverbes [...]. (Argaud \& Kok Escalle $2012: 112$ )

Il s'agit tout d'abord d'un culturel d'ordre ethnologique (ancré sur les modes de vie), mais peu à peu sont intégrés des contenus culturels qui renvoient aux réalités historiques, géographiques et « littéraires ${ }^{5}$ françaises.

Comme le montrent Kok Escalle \& Van Strien-Chardonneau pour la Hollande,

en enseignant la langue, le maître communique d'autres savoirs en particulier la géographie et l'histoire; en témoignent les Dialogues de Parival, les Dialogues françois \& flamends de Mauger ou la Nouvelle grammaire augmentée de Nouveaux Dialogues \& de lettres nouvelles de Marin qui sera imité à la fin du XVIII ${ }^{\mathrm{e}}$ siècle par Cazelles. On trouve également, mais de façon moins systématique que pour la géographie et l'histoire, des dialogues et des exercices où sont dispensés des savoirs scientifiques, sous forme de notions de physique, de sciences naturelles, d'astronomie ou bien des savoirs professionnels concernant la navigation et le commerce. (Kok Escalle \& Van Strien-Chardonneau $2005: 33 / 34,134$ )

6 Cette tendance se poursuit par la disparition progressive des vieilles écoles latines, surtout en Angleterre, en Écosse, en Hollande, et en Allemagne, et leur substitution au XVIII ${ }^{e}$ siècle par des institutions d'enseignement secondaire appelées, par exemple, Realschulen et Gymnasien en Allemagne (Christ 1996 : 18, 70-71; Hammar 1990 et 1991 ; Schröder $1994:$ 14, 188-210; Wakely 1990 ; Weller $1990: 6,103-106)$ où le français est 
introduit tout d'abord comme matière facultative, ainsi que dans les institutions destinées à l'éducation des nobles, comme c'est le cas en Italie, en Espagne et au Portugal notamment, mais aussi dans l'enseignement technique supérieur professionnel dans les Académies des Sciences, Écoles Militaires, Écoles de Chirurgie, Écoles Navales (Fernández Fraile et Suso López 1999: 59-84 ; Lépinette 2000 : 47-78; Pellandra, $1993: 12,32$ et $1998: 6,73-81)$.

7 Par ce biais, la composante fonctionnelle initiale ou communicative, pour utiliser un terme actuel, se double dès le départ d'autres fonctions qui s'y ajoutent au gré des différents contextes d'apprentissage et des apprenants :

le français langue étrangère a été dans l'Europe dès le XVI siècle la langue des négociants internationaux, et aux XVII et $\mathrm{XVIII}^{\mathrm{e}}$ siècles, la langue privilégiée pour la formation de l'esprit, pour la formation de l'honnête homme, pour l'éducation des filles de la bourgeoisie et de l'aristocratie, la langue de la République des Lettres, sans compter son rôle comme langue de la diplomatie. (Argaud \& Kok Escalle 2012:111)

De même, dans le cas de la Hollande :

Lorsque l'on lit les dialogues de Parival [...] ou ceux de Mauger, on voit se dessiner en filigrane le modèle d'éducation aristocratique dont le Grand Tour fait partie intégrante. Le perfectionnement de la langue française qui constituait l'un des objectifs majeurs de ce modèle éducatif, s'inscrit dans un ensemble de pratiques, danse, armes, équitation, musiques, toutes nécessaires pour acquérir l'aisance requise dans la bonne société. Inculquer l'art des bonnes manières et de la conversation [...] est donc aussi l'un des objectifs de ces dialogues [...]. (Kok Escalle \& Van Strien-Chardonneau $2005: 33 / 34: 135$ )

9 Le français remplace ainsi le latin comme langue de la communication internationale (ou « universelle»). Ceci dit, dans l'articulation de la discipline au sein des institutions scolaires qui commencent à offrir le français en tant que matière, deux conceptions s'affrontent au XVIII siècle, comme le constate Minerva pour le cas de l'Italie :

les objectifs que les classes dirigeantes se proposent d'atteindre à travers l'éducation, et en particulier à travers l'enseignement des langues, étaient surtout de caractère mondain. Le français est appris, bien sûr, aussi pour des fins pratiques [...] : la connaissance du français était indispensable pour se tenir au courant des nouveautés scientifiques et littéraires [...]. Mais le français, ne l'oublions pas, fait surtout partie de ces disciplines qui sont enseignées dans un but formatif, disciplines qui préparent l'élève à une conversation mondaine de ton distingué et désinvolte et qui l'initient aux nobles amusements auxquels il est destiné. (Minerva $1992: 9: 8)$

\section{Le français : la langue de la culture, du goût littéraire, du style, de la formation intellectuelle (XVIII ${ }^{\mathrm{e}}$ siècle)}

10 La question - en tant qu'hypothèse de travail - que nous allons examiner est ainsi : pourquoi et comment cette composante culturelle (« littéraire »), présente de manière constante mais subordonnée à l'objectif de formation langagière au XVII siècle, devient-elle centrale et essentielle au XVIII ${ }^{e}$ siècle, au point de rivaliser dans les représentations sociales avec la conception pratique de cette discipline scolaire naissante. bien cette transformation: il justifie dans le prologue de son Arte (1781) l'étude du 
français en tant que matière pratique, nécessaire pour n'importe quelle profession ; en tant qu' "art d'agrément " (Pellandra 1994), "ornement» ou " point d'honneur " (adorno y gala, comme dit Chantreau) pour briller en société, mais surtout en termes de langue universelle, nécessaire pour n'importe quel lettré. Le français est devenu un support de l'éducation de la civilité, et un moyen d'accès à la culture générale, et ceci autant dans les contextes d'enseignement privé que dans les institutions où il est introduit comme matière optionnelle. Ainsi, l'apprentissage du français au sein des institutions scolaires dans plusieurs pays est justifié par sa pertinence sociale d'un point de vue à la fois pratique, économique et culturel (conçu de manière vaste), comme le constate Frijhoff (2009 : 73-93), de la part de la bourgeoisie montante.

Nous ne pouvons, pour des raisons d'espace, que proposer une sélection de cette présence du culturel dans les matériaux d'enseignement édités au XVIII ${ }^{\mathrm{e}}$ siècle dans divers pays européens, et dont disposaient les maîtres et apprenants de français. Les contributions faites au Colloque d'Avila organisé par l'UNED et la SIHFLES en 1997 (Boixareu \& Desné 1999) font état de l'importante présence des auteurs classiques français dans l'enseignement du FLE au XIX ${ }^{e}$ siècle. Mais cette présence du "littéraire " date de bien avant: une place de choix est accordée aux textes littéraires dans l'enseignement du français, dès le XVI ${ }^{e}$ et le XVII ${ }^{e}$ siècles, comme le constatent García Bascuñana \& Santos (2012: 120-130), sous forme d'extraits de textes littéraires recueillis à la fin de l'ouvrage pour leur traduction (version), la pratique des règles de prononciation et l'analyse morphologique. Et cette présence se maintient tout le long du XVIII ${ }^{e}$ siècle ( $c f$. les contributions de Besse, de Bruña, de Fischer, de Medina dans Boixareu \& Desné 1999). El Arte (1781) de Chantreau cité plus haut mérite un relief spécial: le Suplemento qui suit la grammaire contient une sélection de textes (p. 260-304, soit 45 pages!), où l'on trouve un extrait du Télémaque, une description de Madrid, des extraits de l'abrégé d'histoire d'Espagne, un extrait du 'Don Quixote de la Mancha', des extraits de la Gazette d'Amsterdam. Presque quarante textes courts (anecdotes, bons mots, faits historiques) complètent ce Suplemento.

En Allemagne, outre la grammaire de Kramer citée, la Grammaire de Des Pepliers (Leipzig 1717) comprend un « recueil de bons contes et de bons mots, tirés des plus beaux esprits françois de tems » (p. 438-485) ; la première partie des Leçons méthodiques de langue françoise pour Allemands [...], de De Laveaux (1785) « est un recueil de dialogues, scènes de comédies, petits proverbes dramatiques \& autres morceaux originaux françois, ou traductions libres de l'allemand dans le goût de la nation » (Weller 1990: 6, 120). Cet auteur compose également un Cours théorique et pratique de langue et de littérature française (2 volumes, 1784-85), destiné à des apprenants allemands ${ }^{6}$. Dès la fin du XVIII ${ }^{e}$ siècle, les recueils de textes qui sont ajoutés aux grammaires seront publiés de manière autonome et indépendante de la grammaire (ainsi, la Chrestomathie française, Gedike 1792). N'oublions pas finalement que de nombreuses remarques de la partie de syntaxe étaient destinées à l'« intelligence» des bons auteurs, et que les exemples fournis procédaient souvent des textes littéraires.

Dans ce rappel, il faut réserver une place de choix, bien sûr, au Télémaque qui devient un matériel d'enseignement linguistique, littéraire, moral et culturel dans ses multiples supports: en version bilingue, sous forme de textes choisis, sous forme d'enseignements moraux... (Minerva 2003: 30 et 31), outil incontournable pour comprendre l'enseignement du français au XVIII ${ }^{e}$ siècle, et ceci, dans presque tous les pays de l'Europe, et pas uniquement dans les communautés attachées à la religion 
catholique. Il suffit de reprendre ces mots de Hammar : «Le texte le plus utilisé au $18^{\mathrm{e}}$ siècle dans la lecture élémentaire est celui du Télémaque de Fénelon » (1980: 101).

Certains ouvrages vont même plus loin, et insèrent un art poétique dans la grammaire du français : c'est le cas de la Gramática francesa de P.-P. Billet ( $\left.{ }^{2} 1688\right)$, maître de français dans la cour d'Espagne de la fin du XVII et début du XVIII ${ }^{e}$ siècle, qui comprend, dans sa troisième partie, « un Arte poética o breve compendio de la poesía francesa, utilisímo para aprender a conocer, y medir los versos, y a componerlos »; c'est le cas également de El Arte francés (1713), de l'abbé de Vayrac (Suso Lopez à paraître) ${ }^{7}$. Il faut remarquer que les grammaires de français pour étrangers publiées tout au long du XVII ${ }^{e}$ siècle en Europe ( $c f$. Maupas 1632; Cisneros 1635 ; Oudin 1648; Fabre, 1664 ; Berti, 1678, Du Bois de Gomicourt, 1678 ; Chiflet 1680) avaient écarté cette composante de la grammaire, et la prosodie se réduisait à quelques notions insérées dans la partie prononciation et orthographe. La reprise de cette composante de la grammaire (prosodie, art poétique) à la fin du XVII siècle par Billet, puis par de Vayrac, mérite ainsi une mise en relief, et constitue pour nous un «signe » à interpréter. Mais pourquoi introduire la formation du goût poético-littéraire dans l'enseignement du français ?

Selon nous, on ne peut pas régler la question en disant que Billet suit l'exemple de Lancelot, qui avait introduit dans sa Nouvelle méthode pour apprendre facilement la langue latine (1644), une "Breve instruction sur les regles de la Poësie Françoise », à la suite d'un traité sur la poésie latine (Bruña 1999: 24 : 95-115). En effet, les objectifs de ces deux ouvrages ne sont pas comparables : chez Lancelot, il s'agit de jeunes apprenants français, des latinistes, à qui on propose un complément de formation en poésie française ; chez Billet, il s'agit d'apprenants de français étrangers, non encadrés dans une quelconque institution d'éducation, appartenant pour la plupart à la noblesse.

Billet et de Vayrac considèrent que l'apprentissage de la langue française doit incorporer une formation "poético-littéraire»; c'est un trait essentiel de leur conception du français en tant que matière qu'ils enseignent. Nous croyons que ce signe révèle bien une tendance vers la formation «littéraire» des apprenants de français, que nous observons dans l'analyse du contenu des ouvrages signalés.

Ce "littéraire ", il faut le concevoir aux XVII ${ }^{\mathrm{e}}$-XVIII ${ }^{\mathrm{e}}$ siècles comme la "connaissance des lettres, la culture générale " (Caron $1987: 87-103)$. Ce n'est que dans la deuxième moitié du XVIII ${ }^{\text {e }}$ siècle que l'expression « les belles lettres » - qui signifie le savoir fondé sur le livre, la " connaissance des lettres, une culture générale » (TLFi) -, est remplacé par le terme de littérature, et ce terme évolue peu à peu vers la "belle Littérature", "l'agrément, l'ornement, le plaisir, la beauté ", et donc au sens actuel d' "usage esthétique du langage écrit» (Caron 1992: 115). $\mathrm{M}^{\text {me }}$ de Staël définit encore la littérature "dans son acception la plus étendue, c'est-à-dire renfermant en elle les écrits philosophiques et les ouvrages d'imagination, tout ce qui concerne enfin l'exercice de la pensée dans les écrits, les sciences physiques exceptées » $(1800: 29)$.

19 Mais aussi, le «littéraire ", à l'époque, ne représente pas uniquement la littérature consacrée, issue des grands auteurs, idée que soulignent García Bascuñana \& Santos (2012: 120-130). Le Magasin des enfants, le Magasin des adolescents, les Instructions pour les jeunes dames, La nouvelle Clarice, etc., composés ex professo par $\mathrm{M}^{\mathrm{me}}$ Leprince de Beaumont, constituent des exemples de matériaux d'ordre littéraire qui caractérisent également l'enseignement du français de l'époque. Ces ouvrages constituent la base des cours de français dans la 'Société de jeunes dames' qu'elle avait établie à Londres vers 1750-1768 (Fernández Fraile 1999a : 23 : 436-452 ; Uta Janssens $1999: 24: 151-159)$, où la 
langue française était bien plus qu'une habileté langagière; ils montrent bien l'orientation culturelle et éducative que bien des gouvernantes - dont $\mathrm{M}^{\text {lle }}$ Ackermann à la cour de Suède puis en Allemagne (Christ 1987: 819-838) - conféraient à l'enseignement du français pour les filles :

Chaque gouvernante française partant pour l'étranger avait dans sa valise ces volumes précieux [les Magazins de Mme Le Prince de Beaumont], qui en un français très simple et élégant expliquaient d'une façon claire et méthodique toutes les matières dont 'l'éducation domestique' devait meubler les têtes de jeunes filles. Il y avait des textes d'instruction religieuse, des lectures mythologiques, historiques, géographiques, des contes de fées pour les plus petites, les contes moraux pour les adolescentes. En Pologne, comme dans les autres pays, dans chaque château, les jeunes filles étaient élevées selon la méthode de Mme Leprince de Beaumont. (Nikliborg, in Janssens $1999: 34,151$ )

Cette évolution vers une imbrication entre la langue et les réalités culturelles (dont la littérature au sens actuel) susceptibles d'intéresser les apprenants ciblés, en fonction des divers contextes d'enseignement, prend de l'ampleur avec la perte d'influence du latin et l'accès à la culture savante pour de nouvelles couches de la population. Nous n'allons citer que deux exemples. D'un côté, les ouvrages compris sous l'appellation de la «Grammaire des Dames » (Finotti \& Minerva 2012), dont la Grammaire italienne de l'Abbé Antonini (1728), qui servira de modèle aux grammaires postérieures de français. La formule « former l'esprit et le cœur » se complète avec le développement du style et du goût littéraire dans les grammaires de D.-É. Choffin, de M. de Prunay, de N. Adam, de L. Barthélémy..., où sont proposées des contenus culturels de toute sorte ${ }^{8}$ : des textes d'histoire, de géographie, de mythologie, des merveilles du monde, des biographies de dames illustres, des anecdotes tirées de la Gazette de Leyde de l'époque, modèles de composition de lettres - dont la Lettre de $\mathrm{M}^{\text {me }}$ la Marquise Lambert à sa fille -, un recueil de poésies "des meilleurs auteurs", des fables (ainsi une série de Fables de Fénelon et de La Fontaine), les Dialogues des Morts de Fénélon, les Entretiens de $\mathrm{M}^{\mathrm{r}}$ De Callières, des Portraits de La Bruyère...), des chansons, des textes de la littérature gréco-latine, une introduction à la versification française...

21 Un dernier exemple, qu'on oublie habituellement de signaler mais essentiel à nos yeux, dont se fait l'écho Minerva dans son étude sur «Le profil du maître » en Italie (1992: 5-9) : le succès de publications périodiques, qui ont joué le rôle d'outils propagateurs des nouvelles idées politiques, des nouvelles découvertes dans les arts, les sciences et les techniques, y compris la physique et la chimie, des inventions mécaniques et mathématiques, des observations célestes et météorologiques et des découvertes anatomiques 9 , ou encore des controverses artistiques et littéraires. Nous pouvons citer notamment la Gazette, la Gazette de Leyde et la Gazette d'Amsterdam ${ }^{10}$, Le Journal des Savants ${ }^{11}$, L'Année littérair ${ }^{12}$ et les Nouvelles de la République des Lettres et des Arts ${ }^{13}$. Les fonds bibliographiques des Instituts médicaux, botaniques, chirurgicaux, militaires, maritimes, géologiques, etc., qui se créent dans pratiquement tous les pays d'Europe, se remplissent d'ouvrages publiés en français. Le français était la langue de la science, de la philosophie, de la politique, des arts et de la littérature...

Cet ensemble de réalités explicitent, à notre avis, une tendance générale : la formation « littéraire » constitue l'un des objectifs de la discipline «FLE » naissante, aux yeux des maitres/professeurs, aux yeux également des apprenants, constituant une représentation sociale globale qui porte ce savoir au rang des matières d'enseignement, comme cela avait été le cas de l'histoire, des mathématiques ou de la cosmographie. 
Chantreau ajoute à la fin de son Arte un "Choix des livres que tout amateur de la littérature doit se procurer" (ou "Bibliothèque française", 1781: 304-307) ${ }^{14}$. Chantreau pense donc sincèrement que celui qui apprend le français doit forcément être également un "amateur de littérature » et posséder une formation culturelle vaste, d'autant plus s'il se destine à la carrière militaire !

\section{La conception de la langue française (langue maternelle) comme matière d'enseignement}

Nous allons examiner finalement un autre facteur qui va déterminer - en quel degré, il est bien difficile de le préciser - l'évolution de la conception de la " matière FLE », qui va se greffer sur cette représentation que nous avons définie dans la seconde moitié du XVIII ${ }^{\mathrm{e}}$ siècle. Il faut, selon nous, prendre en compte l'évolution même de la conception de la langue française comme matière d'enseignement en tant que langue maternelle ${ }^{15}$, qui détermine des changements dans l'ensemble des collèges, qu'ils soient tenus par les séculiers, par les Jésuites, ou par les autres congrégations enseignantes. La classe des "humanités ${ }^{16}$ " (intermédiaire entre les trois années de grammaire et la classe de rhétorique dans le cursus des collèges), avait tout d'abord pour objet la formation morale :

$\mathrm{Au} \mathrm{XVIII}^{\mathrm{e}}$ et au XIXe siècle, le principal manuel de latin de sixième et de cinquième, le Selectae e profanis scriptoribus historiae, range la plupart des extraits qu'il regroupe dans quatre chapitres intitulés la prudence, la justice, le courage, la modération; et les discours latins ou français que l'élève aura à composer en rhétorique, l'amènent à peu près immanquablement à défendre et à illustrer des "vertus » nous dirions des «valeurs » parmi lesquelles on retrouve à nouveau le courage, la justice, la modération, l'honnêteté, l'abnégation, etc. (Compère \& Chervel 1997 : 74, 10)

Les textes de lecture en latin, choisis en raison de leur vertu formatrice (Ovide, Horace, Homère, Tite-Live, Cicéron...), étaient, eux aussi, des modèles pour les exercices oraux et écrits (mémorisation, analyse, exégèse, commentaire, imitation, composition), où le français gagne progressivement du terrain (Compère \& Chervel 1997 : 74, 17-18); il se crée même un corpus de textes littéraires français qui sont incorporés à la classe des humanités (Racine, Fénelon, La Bruyère, La Fontaine...). La culture de la langue, l'exercice des vertus langagières, deviennent ainsi le lieu et le cœur de l'éducation classique. Y devient ainsi centrale «une formation de l'esprit par la maîtrise du discours " :

qui tend à développer un certain nombre de qualités, la clarté dans la pensée et dans l'expression, la rigueur dans l'enchaînement des idées et des propositions, le souci de la mesure et de l'équilibre, l'adéquation aussi juste que possible de la langue à l'idée (ibid., 1997 : 74, 14).

Cette évolution dans l'«enseignement du français » dans la classe des humanités s'accompagne également de nombreux ouvrages d'ordre pratique concernant la grammaire française, dont ceux de l'abbé Girard (1747), où il déclare : «La PAROLE est la manifestation de la pensée par le secours des mots [...] L'essence du MOT consiste à être une voix prononcée propre à faire naître une idée dans l'esprit » (Premier discours). Girard développe des idées déjà présentes dans la Grammaire de Port-Royal, en y affirmant l'iconicité "entre monde et pensée », et une "isomorphie entre monde, pensée, langage ». Cette conception se base sur la thèse de la «transitivité de la structure du monde à celle de la pensée, et à travers celle-ci, à celle du langage. En 
effet, la segmentation des choses est reflétée dans la segmentation des pensées en idées : à ces idées correspondent, au plan linguistique des mots » (Swiggers $1992: 35$ ). Il faut donc posséder l'outil langagier à la perfection, car «le lien entre le langage et la connaissance du monde est celui que crée la langue » (Bonnet 2001 : en ligne). La considération du français comme langue universelle, ainsi que les arguments avancés par Rivarol et par Schwab ${ }^{17}$, vont renforcer une conception culturelle à l'égard de la langue elle-même et de son enseignement. Dans leurs mémoires, les langues y sont comparées entre elles à partir de l'« image idéale d'une langue parfaite » - c'est-àdire, la clarté dans le transfert de la pensée à l'aide des mots -, ainsi que des « critères souvent politiques et économiques » (Hassler $2005:$ 33/34, 30). Le mythe de la clarté du français, qui dérive de l'ordre naturel (présumé) du français ${ }^{18}$ aura la vie longue (Swiggers 1987 : 75, 5-21). À l'exigence de la pureté de la langue (dans la diction, dans les termes et expressions utilisées) s'ajoute celle de la qualité du discours (sa clarté, son ordre). La culture de la langue, au sens cicéronien de l'expression, devient une partie intrinsèque de l'apprentissage du français, pas uniquement pour les étrangers, mais par les Français autochtones eux-mêmes ${ }^{19}$.

Aussi, les maîtres de français doivent prétendre à un usage distingué de la langue, en se basant sur les écrits des meilleurs auteurs ; aucun maître ne peut se contenter de faire parler à ses élèves un français qui ne soit pas le "français de la nation ", soit le français reconnu par l'Académie française. L'article Langue de l'Encyclopédie s'en prend fortement à la définition de la langue de Frain de Tremblay - à savoir : "ce qu'on appelle langue, est une suite ou un amas de certains sons articulés propres à s'unir ensemble, dont se sert un peuple pour signifier les choses, \& pour se communiquer ses pensées ; mais qui sont indifférens par eux-mêmes à signifier une chose ou une pensée plutôt qu'une autre »- , et défend au contraire qu'on ne peut parler légitimement de langue que dans le cas de la langue nationale, donc, de la langue instituée comme langue rationnelle, sans variétés, pure. Ce n'est donc pas «la suite des sons articulés dont se sert le peuple [français] pour signifier les choses et communiquer ses pensées » que les maitres de langue doivent enseigner à leurs élèves; c'est la seule langue nationale qui mérite le titre de langue.

Nous pouvons envisager que de nombreux maîtres/professeurs de FLE partageaient ces mêmes idées: les publications signalées (Rollin, Batteux, Rivarol, l'Encyclopédie...), éditées en France, étaient disponibles dans la plupart des bibliothèques de l'Europe (en français, ou encore sous forme de traductions), et, certes, certains d'entre eux probablement ceux qui se décidaient à composer une nouvelle grammaire/manuel de français - avaient eu l'occasion de les lire ou les consulter. Une recherche pour montrer dans quelle mesure ce changement dans la conception du FLM se transpose vers le FLE, dans les contenus d'enseignement, dans les démarches de travail, serait à entreprendre, en continuant les travaux proposés et esquissés par Chervel (2009).

Cette conception du français converge, selon nous, avec le même mouvement esquissé auparavant : la conception utilitaire ou pratique de la langue (sa pertinence sociale, où l'essentiel serait d'être capable de comprendre et de communiquer en français dans les usages quotidiens) se déplace vers un au-delà, qui tient tout seul, et la justification logico-culturelle de la matière (langue de culture, langue universelle, langue rationnelle) gagne les institutions où le français commence à être enseigné. 


\section{Conclusion}

Ce survol du contenu culturel présent dans le matériel d'enseignement/apprentissage du FLE édité au XVIII ${ }^{e}$ siècle, d'ordre très divers, comme nous l'avons vu, et à des degrés différents, selon les contextes et les maîtres, nous permet de soutenir que la construction disciplinaire du français dans la seconde moitié du XVIII ${ }^{e}$ siècle met en œuvre une transformation d'un savoir-faire utilitaire ("savoir parler une langue ») et d'un "art d'agrément", qu'on mettait au même rang que la danse, l'escrime ou la musique, en une matière prestigieuse, en lui fournissant un contenu culturel (Pellandra 1994 : 14, 92-101). Nous avons montré, croyons-nous, que l'orientation culturelle de la discipline «FLE » n'attend pas l'institutionnalisation de celle-ci au XIX ${ }^{\mathrm{e}}$ siècle, mais qu'elle est déjà présente dès son introduction comme matière optionnelle dans certaines institutions d'enseignement public, voire dans certains contextes d'enseignement privé. La langue française, dans les représentations sociales, est devenue partout en Europe, au long des $\mathrm{XVII}^{\mathrm{e}}$ et $\mathrm{XVIII}^{\mathrm{e}}$ siècles, un instrument utile, pour les échanges socio-économiques, la condition d'une réussite professionnelle et sociale, un signe de distinction, mais aussi un moyen d'accès à la culture, un outil pour organiser sa pensée et transmettre ses idées, et donc, le meilleur instrument de formation des jeunes. Même dans une première phase de l'apprentissage, le français «se mérite » (pour employer une expression qu'on entend de nos jours), s'obtient avec de l'effort, fuit l'à-peu-près, exige la volonté de bien parler, aide à forger la discipline et à développer l'intellect. La grammaire, bien sûr, devient l'outil essentiel pour acquérir ce français sans fautes.

\section{BIBLIOGRAPHIE}

Sources primaires ${ }^{20}$

BILLET, Pierre Paul (21688). Gramatica francesa, dividida en tres partes. La primera. Contiene los primeros rudimentos [...] La segunda. Comprehende vn tratado muy por extenso de la oracion, ò construccion, mas amplio que el de la primera ediccion, con vn paralelo de la eloquencia española, y francesa, y francesa, $y$ española ;... La tercera. Contiene vn arte poetica, o breve compendio de la poesia francesa [...]. Madrid : Imprenta de Bernardo de Villadiego. Autres éditions : Zaragoza : [s.n.], 1673 ; Amberes : Henrico y Cornelio Verdussen, 1687 ?; Madrid : Juan García Infanzón, ${ }^{3} 1708$.

CHANTREAU, Pedro Nicolás (1781). Arte de hablar bien francés o Gramática completa dividida en tres partes. Madrid : Imprenta de Sancha Rééditions en ${ }^{2} 1786,{ }^{3} 1797$, et nombreuses rééditions et adaptations au XIX ${ }^{\mathrm{e}}$ siècle. En ligne :

[http://bvpb.mcu.es/es/consulta/registro.cmd?id=451063] (05/10/2018).

CHOFFIN, David-Étienne (1761). Nouvelle grammaire à l'usage des Dames et de ceux qui ne savent pas le latin. Berlin : Chez Haude et Spener. Autres éditions en 1757, 1791. 
DES PEPLIERS, J.R. (1717). LA PARFAITE GRAMMAIRE ROYALE Françoise \& Allemande Das ist : Vollkommene Königl. Franß. Teutsche GRAMMATICA [...]. Leipzig: Im Verlag Joh. Ludwig Gleditsch.

GIRARD, Abbé (1747). Les vrais principes de la langue française. Paris : Chez Le Breton.

KRAMER, Matthias (1696). Nouveau Parlement, c'est-à-dire Dialogues François-Alemands. forts plaisans \& même fort courts, pour en faciliter l'intelligence aussi bien que l'imitation à ceux qui commencet à étudier une de ces deux langues. Composez autrefois en François par Monsr. Claude Mauger, natif de Blois, Maître de Langues ; mais présentement à cause de leur pureté \& grande utilité, enrichis de l'Allemand par Matthias Cramer, Maître de Langues. Franckfurt : In Verlegung Johann Baltasar Niesen.

LAVEAUX, Jean-Charles Thibault de (1784-85). Cours théorique et pratique de langue et de littérature française (2 volumes). Berlin : Chez l'auteur et chez Arnold Wever.

(1787) : Leçons méthodiques de langue françoise pour les Allemands : contenant tout ce qui est nécessaire pour apprendre et enseigner cette langue ; à l'usage de l'Université-Caroline de Stouttgard. Stuttgart: Univ.-Caroline.

MAUGER, Claude (1653). The true advancement of the French tongue or a new method and more easie directions for the attaining of it then ever yet have bean published: whereunto are added many choise and select dialogues, containing not onely familiar discourses, but most exact instructions for travell [...] Londres : Martin \& Allestrye.

(111684). Grammaire françoise de Claude Mauger, avec des augmentations [...]. Londres : Printed by R.E. MONFORT, Luis (1815). Principios de Lengua Francesa para uso de los españoles. Valencia : Estevan.

STAËL, Mme de (1800). » Discours préliminaire ». De la littérature considérée dans ses rapports avec les institutions sociales. En ligne : [http://ressources-socius.info/index.php/reeditions/18-reeditionsd-articles/148-discours-preliminaire] (05/10/2018).

VAYRAC, Abad de (1713). El Arte francés, en que van puestas las reglas más acertadas sobre todas las partes de la Oración, para aprehender fácilmente, y con brevedad à leer, pronunciar, escribir, y hablar la Lengua Francesa segun las Decisiones de la Academia Francesa, el uso de la Corte, y el Dictament de los mas celebres Gramaticos. Con un Trato de la Poesia en favor de los que se quisieren dedicar à conocer los Versos, y a componerlos; otro de los Tratamientos que se usan en Francia, y un Formulario para escribir Cartas. $\mathrm{T}$. I. Paris : Pedro Vitte.

Sources secondaires

ARGAUD, Évelyne \& KOK ESCALLE, Marie-Christine (2012). « Le culturel dans l'enseignement du FLE : pratiques didactiques et réflexions de l'historien dans Documents pour l'histoire du français langue étrangère ou seconde ». Le Français dans le Monde, R\&A, 52, 109-119.

BASCUÑANA, Juan Francisco (1999). «L'institutionnalisation du FLE dans l'enseignement public espagnol après la Loi Moyano (1857) : avatars et conséquences ». Documents pour l'Histoire du Français Langue Étrangère ou Seconde, 23, 108-123.

BOIXAREU, Mercedes \& DESNÉ, Roland (Coörd.) (1999). Les auteurs classiques français dans l'enseignement du FLE (18 et $19^{e}$ siècles). Documents pour l'Histoire du Français Langue Étrangère ou Seconde, 24.

BONNET, Valérie (2001). La construction d'une langue savante en Europe du $V^{e}$ au XIX siècle. Thèse de doctorat. Lyon : Université Lumière Lyon 2. En ligne : [http://theses.univ-lyon2.fr/documents/ lyon2/2001/bonnet_v/info] (05/10/2018). 
BRUÑA CUEVAS, Manuel (1996). «La literatura en la enseñanza del francés en España ». In J.

Martínez et al., Aproximaciones diversas al texto literario. Asociación de profesores de filología francesa de la universidad española. Murcia : Universidad.

(1999). « Le fait littéraire dans les manuels de français pour Espagnols au $18^{\mathrm{e}}$ siècle ». Documents pour l'Histoire du Français Langue Étrangère ou Seconde, 24, 95-115.

CARON, Philippe (1987). « Aux origines d'un concept encore flou aujourd'hui, celui de littérature ». In G. Berger \& H.-J. Lüsebrink (eds.), Literaturische Kanonbildung in der Romania. Rheinfeldn : Schaüble.

(1992). Des 'Belles-Lettres' à la 'Littérature', une archéologie des signes du savoir profane en langue française (1680-1760). Louvain/Paris : Peeters.

CERTEAU, Michel de, JULIA, Dominique \& REVEL, Jacques (1975). Une politique de la langue, La Révolution française et les patois. Paris : Gallimard.

CHERVEL, André (2009). « Pour une histoire comparée des disciplines du français langue étrangère et du français langue maternelle ». Le Langage et l'Homme, XXXX.1, 85-98.

CHRIST, Herbert (1987). « Fremdsprachenlehrer im Portrait. Biographisches und Autobiographisches aus 4 Jahrunderten ». In W. Lörscher \& R. Schulze, Perspectives on language in performance. Tübingen: Gunter-Narrr-Verlag, 819-838.

(1996). « L'enseignement du français en Allemagne entre 1648 et 1815 ». Documents pour l'Histoire du Français Langue Étrangère ou Seconde, 18, 63-83.

COMPÈRE, Marie-Madeleine \& CHERVEL, André (1997). « Les humanités dans l'histoire de l'enseignement français ». Histoire de l'éducation, 74, 5-38.

DÜWELL, Henning (1996). «L'universalité de la langue française au $18^{\mathrm{e}}$ siècle vue par un Allemand : Johann Christoph Schwab », Documents pour l'Histoire du Français Langue Étrangère ou Seconde, 18, $42-61$.

FERNÁNDEZ FRAILE, Ma Eugenia (1999a). » Eugénie Foa, sur les traces de Madame de Beaumont ». Documents pour l'Histoire du Français Langue Étrangère ou Seconde, 23, 436-452.

(1999b). « Les textes littéraires comme procédé d'enseignement du FLE dans les Chantreau (1781-1857) ». Documents pour l'Histoire du Français Langue Étrangère ou Seconde, 24, 137-150.

(2005). « Du maître de langues au professeur : parcours sémantique d'une évolution sociale et professionnelle ». Documents pour l'Histoire du Français Langue Étrangère ou Seconde, 33/34, 110-119. FERNÁNDEZ FRAILE, Ma Eugenia \& SUSO LÓPEZ, Javier (1999). La enseñanza del francés en España (1767-1936). Estudio histórico : objetivos, contenidos, procedimientos. Granada : Método.

FinOTTI, Irene \& MinERVA, Nadia (2012). Voix féminines. Ève et les langues dans l'Europe moderne. Documents pour l'Histoire du Français Langue Étrangère ou Seconde, 47/48.

FRIJHOFF, Willem (2009). «L'enseignement secondaire : un concept opératoire pour l'Europe moderne?». Histoire de l'éducation, 124, 73-93.

GARCÍA BASCUÑANA, Juan \& SANTOS, Ana Clara (2012). » Le texte littéraire dans l'enseignement du FLE : histoire, variations et perspectives ». Le Français dans le Monde, R\&A, 52, 120-130.

HAMMAR, Elisabet (1980). L'enseignement du français en Suède jusqu'en 1807, méthodes et manuels. Stockholm : Akademilitteratur. 
(1990). « Histoire de l'enseignement du français en Suède. Résultats et problèmes de la recherche ». Documents pour l'Histoire du Français Langue Étrangère ou Seconde, 6, 63-72.

(1991). La « Française ». Mille et une façons d'apprendre le Français en Suède avant 1807. Stockholm : Almqvist \& Wiksell International.

HASSLER, Gerda (2005). « Les maîtres de langues et la constitution de la philologie romane en Allemagne ». Documents pour l'Histoire du Français Langue Étrangère ou Seconde, 33/34, 22-46.

JANSSENS, Uta (1999). « Les Magazins de Mme Leprince de Beaumont et renseignement privé et public du français en Europe (1750-1850) ». Documents pour l'Histoire du Français Langue Étrangère ou Seconde, 24, 151-159.

KOK ESCALLE, Marie-Christine \& VAN STRIEN-CHARDONNEAU, Madeleine (2005). « Apprentissage de la langue et comparatisme culturel en Hollande : le métier de maître de langue (XVII ${ }^{\mathrm{e}}-\mathrm{XIX}^{\mathrm{e}}$ siècles ». Documents pour l'Histoire du Français Langue Étrangère ou Seconde, 33/34, 120-143.

LÉPINETTE, Brigitte (2000). L'enseignement du français en Espagne au XVIII siècle dans ses grammaires : contexte historique, concepts linguistiques et pédagogie. Münster : Nodus.

MINERVA, Nadia (1992). « Le profil du maître à travers les dialogues et les lettres des manuels de français à l'usage des Italiens (XVII et XVIII ${ }^{\mathrm{e}}$ siècles) ». Documents pour l'Histoire du Français Langue Étrangère ou Seconde, 9, 5-9.

MINERVA, Nadia (dir.) 2003. Les Aventures de Télémaque. Trois siècles d'enseignement du français. Documents pour l'Histoire du Français Langue Étrangère ou Seconde, I, 30 et II, 31.

PELLANDRA, Carla (1993). «Que lisait-on dans les classes de français d'autrefois ? Les contenus culturels de quelques manuels pour l'enseignement du français publiés en Italie de 1846 à 1908 ». Documents pour l'Histoire du Français Langue Étrangère ou Seconde, 12, 32-38.

(1994). « La scolarisation d'un art d'agrément : l'enseignement du français en Italie au XIX ${ }^{\mathrm{e}}$ siècle ", Documents pour l'Histoire du Français Langue Étrangère ou Seconde, 14, 92-101.

(1998). «L'enseignement du français dans quelques collèges de jésuites du XVII ${ }^{\mathrm{e}}$ au XVIII ${ }^{\mathrm{e}}$ siècle ». Documents pour l'Histoire du Français Langue Étrangère ou Seconde, 6, 73-81.

SCHRÖDER, Konrad (1994). « Französischunterricht in Berlin im 18. Jahrundert ». Documents pour l'Histoire du Français Langue Étrangère ou Seconde, 14, 188-210.

SUSO LÓPEZ, Javier (1999). « Littérature française et formation esthétique et morale dans les manuels de français de la fin du XIX ${ }^{\mathrm{e}}$ siècle ». Documents pour l'Histoire du Français Langue Étrangère ou Seconde, 24, 205-221.

(2018, à paraître). « Éthique professionnelle et disciplinarisation du français langue étrangère : quelques exemples en territoire allemand ».

SWIGGERS, Pierre (1987). « À l'ombre de la clarté française ». Langue française, 75, 5-21.

(1992). Abbé Gabriel Girard. Les vrais principes de la langue française. Paris : Droz.

(2009). « Enseignement et apprentissage des langues vernaculaires à l'aube des Temps modernes. Attentes sociales et réponses didactiques ». Le Langage et l'Homme, XXXXIV, 1, 99-109.

WAKELY, Richard (1990). « Aspects de l'enseignement du français en Écosse avant 1800 ». Documents pour l'Histoire du Français Langue Étrangère ou Seconde, 6, 82-102.

WELLER, Franz-Rudolf (1990). «L'enseignement du français en Allemagne à la veille de la Révolution française ». Documents pour l'Histoire du Français Langue Étrangère ou Seconde, 6, 103-125. 


\section{NOTES}

1. Nous utilisons la lexie 'français langue étrangère' (désormais FLE) pour désigner l'ensemble des réalisations et variantes linguistiques, changeantes diachroniquement, sous lesquelles la langue française était enseignée en dehors de la France, dans les divers contextes d'enseignement et d'apprentissage.

2. Nous limitons toutefois notre étude aux XVII ${ }^{e}$ et XVIII ${ }^{e}$ siècles, où l'enseignement du français garde encore une certaine homogénéité.

3. Pensons à la prolifération de colloques/dialogues plurilingues ( $c f$. Berlaimont) qui se produit au XVI ${ }^{\mathrm{e}}$ siècle, dans les principales langues de l'Europe, et qui continue au XVII ${ }^{\mathrm{e}}$ siècle (ainsi, Antoine Oudin publie en 1650 une version de ces dialogues en quatre langues).

4. Nous citons la onzième édition, de 1684. Cette grammaire connaît de nombreuses éditions au XVII ${ }^{\mathrm{e}}$ siècle, à Londres (181698), mais aussi à la Haye (1675) et à Bruxelles (1696) - éditions auxquelles collabore également P. Festeau -, et également à Bordeaux (1689, chez Simon Boe). Les dialogues contenus dans cette grammaire seront repris par Matthias Kramer (1696) et jouiront d'un grand succès en Allemagne tout au long du XVIII ${ }^{\mathrm{e}}$ siècle.

5. Nous revenons plus loin sur la conception du « littéraire » aux XVII - XVIII ${ }^{\mathrm{e}}$ siècles.

6. Dans cette étude nous posons que «De Laveaux partage une vision du français comme une langue unique (sans variations, fixée une fois pour toutes dans les moules du classicisme), élitaire, mais à la fois universelle, langue de la culture littéraire et de la formation intellectuelle ».

7. Nous nous limitons ici à citer des exemples présents dans l'enseignement du FLE en Espagne. D'autres cas seraient à signaler, ainsi : les Principios de Lengua Francesa [...], de L. Monfort (1815), ouvrage qui accorde également une place, quoique minimale, à la versification (15-17).

8. Le tome II de la Nouvelle Grammaire de Choffin (1761) se compose ainsi de 416 pages destinées à ces contenus culturels, ou «Diverses pièces qui ont paru propres à former le style, l'esprit et le cœur».

9. Il ne faudrait pas dédaigner non plus à cet égard le rôle de L'Encyclopédie de d'Alembert de Diderot.

10. La Gazette est créée en 1631 avec l'appui de Richelieu par Théophraste Renaud, qui informait sur des nouvelles politiques ou diplomatiques provenant de l'étranger ou de la Cour. La Gazette de Leyde a été publiée entre 1677 et 1811 : ce journal est l'un des plus lus et des plus influents de la seconde moitié du XVIII e siècle. La Gazette d'Amsterdam était une source essentielle d'informations politiques et paraissait deux fois par semaine.

11. Paru en 1665, hebdomadaire irrégulier jusqu'en 1723, mensuel jusqu'en 1792, rétabli en 1816.

12. Périodique littéraire français (créé en 1754 à Paris, par Élie Fréron), qui paraissait par cahiers tous les dix jours, et qui eut un succès immédiat.

13. Ouvrage périodique dont le principal rédacteur est $\mathrm{M}$. Pahin de Champlain de La Blancherie (Paris, Ruault, 1777), qui comporte des informations diverses sur la littérature, les sciences, les beaux-arts, les techniques, tout ce qui peut intéresser l'ensemble des personnes cultivées.

14. Ce choix comprend des ouvrages visant la formation linguistique (la Grammaire de Wailly, les Synonymes de l'abbé Girard), la formation intellectuelle et morale (ouvrages de logique, de morale, de mythologie), des éléments de formation géographique (moderne, générale, livres de voyages), et historique (histoire ancienne, romaine, universelle, de l'Espagne, du Portugal, de la France, de l'Angleterre), de formation scientifique (physique et histoire naturelle) et de formation esthétique et littéraire. $C f$. Fernández Fraile (1999b) pour le détail.

15. Telle qu'elle se dessine notamment à travers les ouvrages de Ch. Rollin (1732, De la manière d'enseigner et d'étudier les belles lettres, dont le projet est de «former le goût en enseignant les langues ») et de Charles Batteux (Traité des beaux-arts en général ou les Beaux-arts réduits à un même 
principe, 1746 ; Cours de belles-lettres distribué par exercices, 1747-1748, réintitulé Cours de belleslettres ou Principes de littérature, 1753).

16. «On appelle humanités ce qu'on apprend ordinairement dans les collèges jusqu'à la philosophie exclusivement » (Dictionnaire de l'Académie française, ${ }^{4} 1762$ ).

17. Le prix est accordé en 1784 ex-aequo aux mémoires de ces deux auteurs (H. Düwell 1996 : 18, 42-61). Rappelons que le concours organisé par l'Académie Royale des Sciences et Belles-Lettres de Prusse ne posait pas la question de savoir quelle langue méritait le titre de langue universelle, mais plutôt : « Qu'est-ce qui a rendu la langue Française universelle ? Pourquoi mérite-t-elle cette prérogative ? Est-il à présumer qu'elle la conserve? ».

18. Cf. à cet égard l'article Langue dans l'Encyclopédie, rédigé par Beauzée.

19. N'oublions pas que seulement $10 \%$ de la population parlait français à la veille de la Révolution française (cf. l'enquête de l'Abbé Grégoire, in Michel de Certeau et al, 1975).

20. Pour des raisons d'espace, nous incorporons ici uniquement les références primaires qui ont fait l'objet d'un travail d'analyse, et laissons de côté les ouvrages qui n'ont eu qu'une mention du seul titre, étant toutefois faciles à retrouver dans les nombreux catalogues existants.

\section{RÉSUMÉS}

Nous analysons dans cet article quelles étaient les idées force quant à la représentation du français qui sont présentes aux XVII $^{\mathrm{e}}$ et XVIII ${ }^{\mathrm{e}}$ siècles, au début du processus de la disciplinarisation et de l'institutionnalisation du français langue étrangère (FLE) en tant que matière scolaire. La conception du FLE comme un savoir-faire utilitaire et comme un «art d'agrément " s'enrichit d'une composante culturelle et formative grâce auxquelles elle acquiert le statut de langue de culture et de formation intellectuelle, et donc, susceptible de devenir une matière scolaire prestigieuse. Ce statut devient la représentation dominante de la discipline auprès du corps professoral et dans l'ensemble des divers pays européens.

We analyze in this article the main ideas as for the representation of French which are present along the $17^{\text {th }}$ and $18^{\text {th }}$ centuries, at the beginning of the process of the disciplinarisation and the institutionalization of the French as a foreign language (FLE) as school subject. The conception of FLE as a utilitarian know-how and as an enjoyment grows rich of a cultural and formative component, and so it acquires the status of language of culture and intellectual training, susceptible to become a prestigious school subject. This status becomes the dominant representation of the discipline to the teaching staff and in the whole of the diverse European countries.

\section{INDEX}

Keywords : teaching French, culture, disciplinarisation, institutionalization, conception of French language, representations of French language

Mots-clés : enseignement du français, culture, disciplinarisation, institutionnalisation, conception du français, représentations du français 
AUTEUR

JAVIER SUSO LÓPEZ

Universidad de Granada 